\title{
A GENERALIZED HÖLDER INEQUALITY AND A GENERALIZED SZEGO THEOREM
}

\author{
FLORIN AVRAM AND LAWRENCE BROWN \\ (Communicated by William D. Sudderth)
}

\begin{abstract}
We prove a limit theorem connected to graphs, which when the graph is a cycle reduces to Szego's theorem for the trace of a product of Toeplitz matrices. The main tool used is a Hölder type inequality for multiple integrals of functions which are applied to variables satisfying linear dependency relations.
\end{abstract}

\section{A. INTRODUCTION}

When establishing the convergence of the distribution of sums of functions of Gaussian fields, by the method of moments, one is led to the study of the asymptotic behaviour of a certain type of deterministic sums associated with graphs (see Definition (1.1) below). For example, these sums are encountered in the work of Giraitis and Surgailis [G1] and [G2], and Fox and Taqqu [F1] and [F2].

When the graph is a cycle, these sums reduce to the trace of a product of Toeplitz matrices, first studied by Grenander and Szego [GS]. We show that a Szego type result holds also in the case of general graphs. The key step in establishing it was showing that a generalized Hölder inequality, of independent interest, holds under the "power counting" conditions known to physicists.

In a companion paper [A2] we show how our results, combined with graph theoretic results, can be used for establishing convergence to the Gaussian distribution, by the method of moments.

Definitions. Let $G=(\mathscr{V}, \mathscr{E})$ be a directed graph, with $V$ vertices, $E$ edges, and $\mu$ components.

Received by the editors August 31, 1987 and, in revised forms, December 16, 1988 and January 26, 1989.

1980 Mathematics Subject Classification (1985 Revision). Primary 26D15; Secondary 94C15.

Key words and phrases. Generalized Hölder inequality, power counting conditions, graph sums, polymatroid, bond matroid.

Work partly supported by the U. S. Army Research Office through the Mathematical Science Institute of Cornell University. 
Associated with every edge $e, e=1, \ldots, E$, there is a function $f^{(e)}(\chi) \in$ $\mathscr{L}_{p_{e}}[-\pi, \pi]$, extended periodically to the whole line; here,

$$
\mathscr{L}_{p}=\left(\begin{array}{rl}
L_{p} & \text { for } 1 \leq p<\infty \\
C & \text { for } p=\infty
\end{array}\right.
$$

The Fourier coefficients of $f^{(e)}(\chi)$ are denoted by

$$
f_{k}^{(e)}:=\int_{-\pi}^{\pi} \exp (i k \chi) f^{(e)}(\chi) \frac{d \chi}{2 \pi} .
$$

Associated with each vertex $v, v=1, \ldots, V$, there is an index $j_{v}, j_{v}=$ $1,2, \ldots$ We consider sums of the form

$$
S_{n}(G)=\sum_{j_{1}, \ldots, j_{V}=1}^{n} \prod_{e=1}^{E} f_{j_{v_{e}, 1}-j_{v_{e}, 0}}^{()^{\prime}},
$$

where $v_{e, 1}$ and $v_{e, 0}$ are the end and start vertices of the edge $e$.

When the graph $G$ is a cycle, $S_{n}(G)$ is the trace of a product of Toeplitz matrices. Genander and Szego [GS] showed in this case that when $f^{(e)}(\chi) \in$ $\mathscr{L}_{\infty}(=C)$, then:

$$
\frac{S_{n}}{n} \longrightarrow \int_{-\pi}^{\pi} \prod_{e=1}^{E} f^{(e)}(\chi) \frac{d \chi}{2 \pi}
$$

Let $Z_{e}=\left(p_{e}\right)^{-1}$.

By Hölder's inequality, the condition to ensure that the R.H.S. of (1.2) is well defined is:

$$
\sum_{e=1}^{E} Z_{e} \leq 1
$$

In fact, (1.3) ensures also that (1.2) holds (see [A]). Note also that when $n=2$, (1.2) becomes Parseval's relations (see Katznelson [K, pg. 35]).

These results for cycle graphs have analogues in the case of general graphs. The R.H.S. of (1.2) becomes then a multiple integral with respect to variables $y_{c}, c=1, \ldots, C$, where $C$ is the maximal number of independent cycles of the graph.

$$
\frac{S_{n}(G)}{n^{\mu}} \longrightarrow I(G)=\int_{[-\pi, \pi]^{C}} \prod_{e=1}^{E} f^{(e)}\left(X_{e}\right) \prod_{c=1}^{C} \frac{d y_{c}}{2 \pi} .
$$

Here, $\mu$ is the number of components of the graph, and $X_{e}$ are certain linear combinations of the $y_{c}$ 's (see (1.5)). The condition (1.3) has to be replaced then by conditions which ensure that $I(G)$ is well defined. 


\section{B. THE HöLDER INEQUALITY FOR LINEARLY DEPENDENT VARIABLES}

Let $X_{i}, i=1, \ldots, n$, be variables which are linear combinations of the variables $y_{j}, j=1, \ldots, m$,

$$
X_{i}=\sum_{j=1}^{m} y_{j}\left[y_{j}: X_{i}\right]
$$

with coefficients $\left[y_{j}: X_{i}\right]$ which are integers. The $X_{i}$ 's will be viewed also as the column vectors in $\mathbf{R}^{m}$ with components $\left[y_{j}: X_{i}\right], j=1, \ldots, m$. For any subset $A \subset\left\{X_{1}, \ldots, X_{n}\right\}$, we denote by $r(A)$ the rank of $A \in \mathbf{R}^{m}$.

For each $i=1, \ldots, n$, we have a function $f^{(i)}(X) \in L_{p_{i}}[-\pi, \pi]$. Let $Z_{i}=$ $\left(p_{i}\right)^{-1}$. We will say that $\left(Z_{i}\right)$ and $\left(X_{i}\right)$ satisfy the power counting conditions (P.C.) if:

$$
\sum_{X_{i} \in A} Z_{i} \leq r(A), \quad \forall A \subset\left\{X_{1}, \ldots, X_{n}\right\}
$$

Theorem 1. Suppose that for $i=1, \ldots, n, f^{(i)}(X) \in L_{p_{i}}, 1 \leq p_{i} \leq \infty$, that $X_{i}, i=1, \ldots, n$, are linear combinations with integer coefficients of $y_{j}, j=$ $1, \ldots, m$, and that the power counting conditions for $\left(p_{i}\right)^{-1}$ and $X_{i}$ hold. Then,

$$
\left|\int_{[-\pi, \pi]^{m}} \prod_{i=1}^{n} f^{(i)}\left(X_{i}\right) \prod_{j=1}^{m} \frac{d y_{j}}{2 \pi}\right| \leq \prod_{i=1}^{n}\left\|f^{(i)}\right\|_{p_{i}} .
$$

Remarks. 1. For an independent set $A$ the (P.C.) condition follows automatically from $Z_{i} \leq 1$. It is enough to check the (P.C.) condition for dependent sets $A$, and in fact only for "maximal" ones, i.e., sets so that $X_{i}$ dependent of $A$ implies $X_{i} \in A$.

2. The conditions (P.C.) have been know for a long time to ensure the convergence of the L.H.S. of (1.4), at least in the case when the functions $f^{(e)}(X)$ are regularly varying, and they were called the "power counting" conditions. See for example Weinberg [WG], Lowenstein and Zimmerman [LZ], Manoukian [M], Fox and Taqqu [F2].

Theorem 1 is proved in $\S 2$.

It would be interesting to extend Theorem 1 so that we are allowed to have in the L.H.S. of (1.4) functions of several variables $f^{(i)}\left(X_{i, 1}, \ldots, X_{i, k_{i}}\right), i=$ $1, \ldots n$.

We provide in this direction a partial result, which states basically that Theorem 1 continues to hold as long as the functions $f^{(i)}$ can be approximated in a very strong sense by sums of products.

Let us denote now by $\bar{L}_{p}\left([-\pi, \pi]^{k}\right)=L_{p} \otimes_{\gamma} \cdots \otimes_{\gamma} L_{p}$ the tensor product of $L_{p}, \ldots, L_{p}$ ( $k$ times), endowed with the greatest cross norm (i.e., for a finite 
sum of products:

$$
h\left(X_{1}, \ldots, X_{k}\right)=\sum_{i=1}^{S} \prod_{j=1}^{k} f^{(i, j)}\left(X_{j}\right)
$$

we define the norm

$$
\||h|\|_{p}=\inf \sum_{i=1}^{S} \prod_{j=1}^{k}\left\|f^{(i, j)}\right\|_{p}
$$

where the infimum is taken over all decompositions of $h$ of the form above; completing then the set of finite sums of products under this norm yields the tensor product space $\bar{L}_{p}\left([-\pi, \pi]^{k}\right)$; see for example Light and Cheney [LC, page 6]).

Note that \|\|$_{p} \leq\|\|\|\|_{p}$, and thus $\bar{L}_{p}$ is only a subspace of $L_{p}$

Theorem $1^{\prime}$. Suppose that for $i=1, \ldots, n, f^{(i)} \in \bar{L}_{p_{i}}\left([-\pi, \pi]^{k_{i}}\right)$, and that $X_{1,1}, \ldots, X_{1, k_{1}}, \ldots, X_{n, 1}, \ldots, X_{n, k_{n}}$ are linear combinations with integer coefficients of $y_{j}, j=1, \ldots, m$, taken modulo $2 \pi$. To each variable $X_{i, j}$ we associate a number $Z_{i, j}=\left(p_{i}\right)^{-1}$. Then, if the power counting conditions

$$
\sum_{X_{i, j} \in A} Z_{i, j} \leq r(A), \quad \forall A \subset\left\{X_{i, j}, i=1, \ldots, n, j=1, \ldots, k_{i}\right\}
$$

are satisfied, the Hölder inequality

$$
\left|\int_{[-\pi, \pi]^{m}} \prod_{i=1}^{n} f^{(i)}\left(X_{i, 1}, \ldots, X_{i, k_{i}}\right) \prod_{j=1}^{m} \frac{d y_{j}}{2 \pi}\right| \leq \prod_{i=1}^{n}\left\|\left|f^{(i)}\right|\right\|_{p_{i}}
$$

holds.

Remark. $\left(1.4^{\prime}\right)$ does not hold if we replace $\bar{L}_{p_{i}}$ by the larger spaces $L_{p_{i}}$ and the greatest cross norms by the smaller \|\|$_{p}$ norms. Indeed suppose it would, and let us take $f^{(1)}(X)=(X)^{-1+\varepsilon} \in L_{1}[-\pi, \pi]$, and $f^{(2)}\left(X_{1}, X_{2}\right) \in L_{2}\left([-\pi, \pi]^{2}\right)$, and consider:

$$
\int_{[-\pi, \pi]^{2}} f^{(1)}\left(X_{1}+X_{2}\right) f^{(2)}\left(X_{1}, X_{2}\right) d X_{1} d X_{2} \text {. }
$$

Since the power counting conditions here are satisfied, we would get that the integral above is finite for any $f^{(2)} \in L_{2}\left([-\pi, \pi]^{2}\right)$ which in turn would imply that $f^{(1)}\left(X_{1}+X_{2}\right) \in L_{2}\left([-\pi, \pi]^{2}\right)$; however, that is easily checked to be false if $\varepsilon<1 / 2$, leading thus to a contradiction.

Proof. Theorem $1^{\prime}$ follows immediately from Theorem 1 and the definition of the greatest cross norm $\|||\|_{p}$.

\section{SZEGo'S THEOREM FOR GRAPHS}

Definition. Let $F$ denote a spanning forest of the graph $G$ and $F^{\prime}$ its complement, i.e., the edges of the graph not belonging to $F$. For each edge $e \in F^{\prime}$ 
there is a unique cycle $c$ such that $c \cap F^{\prime}=e$. This system of $C$ cycles $(C=E-V+\mu)$ is called the fundamental set of cycles associated to $F^{\prime}$.

With each cycle $c$ of a fundamental set we associate a variable $y_{c}$. Let then

$$
X_{e}=\sum_{c=1}^{C} y_{c}[c: e],
$$

where each cycle is assigned an arbitrary orientation and

$$
[c: e]=\left(\begin{array}{rl}
0 & \text { if } e \notin c, \\
1 & \text { if } e \in c, \text { and their directions coincide, } \\
-1 & \text { if } e \in c, \text { and the directions do not coincide. }
\end{array}\right.
$$

Theorem 2. Let $G$ be a graph with $\mu$ connected components. Suppose that for $e=1, \ldots, E, f^{(e)}(\chi) \in \mathscr{L}_{p_{e}}, 1 \leq p_{e} \leq \infty \quad\left(\mathscr{L}_{\infty}=C\right)$, and that

$$
\sum_{e \in A} Z_{e} \leq r(A), \quad \forall A \subset \mathscr{C},
$$

where $r(A)$ is the rank of the $\left\{X_{e}\right\}_{e \in A}$, interpreted as vectors over $\mathbf{R}^{C}$, with coefficients given by (1.5). Then

$$
\frac{1}{n^{\mu}} S_{n}(G) \longrightarrow I(G)=\int_{[-\pi, \pi] c} \prod_{e=1}^{E} f^{(e)}\left(X_{e}\right) \prod_{c=1}^{C} \frac{d y_{c}}{d \pi},
$$

where $y_{c}$ can correspond to any set of fundamental cycles.

Theorem 2 is proved in $\S 3$.

Remarks. 1. By Theorem 1, the conditions (1.7) are precisely the necessary conditions which ensure that the R.H.S. of (1.8) is well defined.

2 . It is well known in graph theory that a subset $A$ of the column vectors $[c: e], c=1, \ldots, C$, is dependent iff the removal of the edges in $A$ increases the number of connected components of the graph (see Bondy and Murty, [BM, Theorem 12.2ii]). This dependence structure is thus independent of the particular choice of the maximal set of independent cycles, and is known under the name of the bond (or cutset) matroid of the graph; its rank function is also known (see Bryant and Perfect, [BP, Exercise 3.3 and page 125]):

$$
r(A)=|A|+\mu-c(A),
$$

where $c(A)$ denotes the number of connected components of the subgraph $(\mathscr{V}, A)$.

\section{Proof of Theorem 1}

It is enough to check that (1.4) holds for the extremal points of the convex domain determined by the (P.C.) conditions, since then it must hold for all the domain by Riesz-Thorin interpolation (see, for example, Bergh and Lofstrom, [BL, Exercise 1.13]). 
Furthermore the extremal points of the (P.C.) domain have only coordinates which equal 0 or 1 . In fact, polytopes determined by the same special type of constraints as the (P.C.) domain are called polymatroids (see Welsh, 18.3, Theorem 1), and formulas for their coordinates are known explicitly. (See Welsh [W, 18.4, Theorem 1].)

Let now $\mathbf{z}=\left(Z_{1}, \ldots, Z_{n}\right)$ be a point whose coordinates are all 0 or 1 , and let $A_{\mathrm{z}}$ be the subset of $X_{i}, i=1, \ldots, n$, corresponding to its 1 coordinates. Note that $\mathbf{z}$ satisfies the (P.C.) conditions iff $A_{\mathbf{z}}$, interpreted as a set of vectors in $\mathbf{R}^{m}$, is independent.

Indeed,

$$
\begin{aligned}
\sum_{i \in A} Z_{i} \leq r(A), \quad \forall A & \leftrightarrow \sum_{i \in A} Z_{i} \leq r(A), \quad \forall A \subset A_{\mathbf{z}} \\
& \leftrightarrow \operatorname{card}(A)=r(A), \quad \forall A \subset A_{\mathbf{z}} \\
& \leftrightarrow A_{\mathbf{z}} \text { independent. }
\end{aligned}
$$

It remains now only to show that Hölder's inequality holds when $A_{\mathbf{z}}$ is independent. W.1.o.g., we may assume that the complement of $A_{\mathrm{z}}$ is void (the bounds of the bounded functions can be taken out of the integral). Let us switch now to a new set of variables $u_{j}$, which includes those $X_{i}$ with $i \in A_{z}$ and so that each $u_{j}$ is a linear combination with integer coefficients of the $y_{j}$. This last condition ensures that the Jacobian is cancelled by the "wrapping around of the torus," i.e.:

$$
\int \prod_{i \in A_{\mathbf{z}}} f^{(i)}\left(X_{i}\right) \prod_{j=1}^{m} d y_{j}=\int \prod_{i \in A_{\mathbf{z}}} f^{(i)}\left(u_{i}\right) \prod_{j=1}^{m} d u_{j} .
$$

But now each $f^{(i)}$ can be integrated separately, and the result holds.

\section{Proof of Theorem 2}

For any vertex $v$ and edge $e$, let

$$
[v, e]=\left(\begin{array}{rl}
0 & \text { if } v, e \text { are not incident } \\
1 & \text { if } v \text { is the end point of } e \\
-1 & \text { if } v \text { is the start point of } e
\end{array}\right.
$$

Thus, $j_{v_{e}, 1}-j_{v_{e, 0}}=\sum_{v=1}^{V} j_{v}[v: e]$. Let now

$$
u_{n}=\sum_{e}[v: e] \chi_{e} \text {. }
$$

Lemma 1.

$$
S_{n}(G)=\int_{[-\pi, \pi]^{E}} \prod_{v=1}^{V} \Delta_{n}\left(u_{n}\right) \prod_{e=1}^{E}\left(f^{(e)}\left(\chi_{e}\right) \frac{d \chi_{e}}{2 \pi}\right),
$$

where $\Delta_{n}(\chi)=\sum_{k=1}^{n} e^{i k \chi}$, and $u_{v}$ are linear combinations of $\chi_{e}$ defined in (3.1). 
Proof. Replace in $S_{n}$ each $f_{k}^{(e)}$ by $\int_{-\pi}^{\pi} \exp \left(i k \chi_{e}\right) f^{(e)}\left(\chi_{e}\right) d \chi_{e} / 2 \pi$. Thus,

$$
\begin{aligned}
S_{n} & =\sum_{\substack{j_{v=1} \\
v=1, \ldots, V}}^{n} \int \prod_{e=1}^{E} f^{(e)}\left(\chi_{e}\right) \exp \left(i \sum_{e, v} j_{v}[v: e] \chi_{e}\right) \prod_{e=1}^{E} \frac{d \chi_{e}}{2 \pi} \\
& =\int \prod_{v=1}^{V} \Delta_{n}\left(\sum_{e}[v: e] \chi_{e}\right) \prod_{e=1}^{E} f^{(e)}\left(\chi_{e}\right) \prod_{e=1}^{E} \frac{d \chi_{e}}{2 \pi} \\
& =\int \prod_{v=1}^{V} \Delta_{n}\left(u_{v}\right) \prod_{e=1}^{E}\left(f^{(e)}\left(\chi_{e}\right) \frac{d \chi_{e}}{2 \pi}\right) .
\end{aligned}
$$

Proof of Theorem 2. It is clearly enough to establish the case $\mu=1$, of connected graphs.

The idea of the proof is to integrate in (3.2) first over the complement of the space generated by the $u_{v}$ 's (and as it is well known in graph theory, the orthogonal of the vertex variables $u_{v}$ are the cycle variables $y_{c}$ ). It turns out that the resulting function is continuous in $u_{v}$, and the measures $1 / n \prod_{v=1}^{V} \Delta_{n}\left(u_{v}\right)$ converge weakly to the $\delta_{0}$ measure, yielding thus the limit $I(G)$.

Let now $T$ denote some tree of the graph, and $T^{\prime}$ its complement. By reordering the edges, we can assume that the edges in $T^{\prime}$ are $e=1, \ldots, C$. We will change variables in (3.2), choosing as new variables $y_{c}=\chi_{c}, c=1, \ldots C$, and $u_{v}, v=1, \ldots, V-1\left(u_{V}=-\sum_{v=1}^{V-1} u_{v}\right)$. Let $M$ denote the matrix with elements $M_{v, e}=[v: e], v=1, \ldots, V-1, e=1, \ldots, E$, let $A$ denote its last minor of order $V-1$, and $B$ denote its first $C$ columns. Thus the transformation is

$$
\left(y_{1}, \ldots, y_{C}, u_{1}, \ldots, u_{V-1}\right)^{t}=\left(\begin{array}{cc}
I_{C} & 0 \\
B & A
\end{array}\right)\left(\chi_{1}, \ldots, \chi_{E}\right)^{t} .
$$

To determine the inverse transformation, we will use two well-known facts in graph theory:

(a) $A$ is nonsingular, and in fact $|\operatorname{det}(A)|=1$ (see [N, Theorem 3.3]).

(b) If we let $M_{c, e}^{*}=[c: e]$, for $c \in \mathscr{C}, e \in \mathscr{E}$, where $\mathscr{C}$ is any fundamental set of cycles, arbitrarily oriented, then $M^{*} M^{t}=0$. If we associate now to each edge $e \in T^{\prime}$ the unique cycle $c$ for which $c \cap T^{\prime}=e$, we obtain a fundamental set $\mathscr{C}$ of cycles (see [N, Theorem 2.22]); assigning each $c \in \mathscr{C}$ the orientation of its corresponding edge will yield a matrix $M^{*}$ of the form $M^{*}=\left(I_{c} D\right)$. By the above orthogonality of $M^{*}$ and $M$, it follows that $B+A D^{\prime}=0$.

Using (a) and (b), it is now easy to check that the inverse of the transformation (3.3) is:

$$
\left(\chi_{1}, \ldots, \chi_{E}\right)^{t}=\left(\begin{array}{cc}
I_{C} & 0 \\
D^{t} & A^{-1}
\end{array}\right)\left(y_{1}, \ldots, u_{V-1}\right)^{t}=\left(\left(M^{*}\right)^{t} N^{t}\right)\left(y_{1}, \ldots, u_{V-1}\right)^{t},
$$

where we denoted by $N^{t}$ the last $V-1$ columns of the matrix. 
Since the matrix in (3.3) has integer entries, (3.2) becomes, after changing to the new variables and integrating the $y_{c}$ 's,

$$
S_{n}=\int_{[-\pi, \pi]^{v-1}} \Delta_{n}\left(-\sum_{v=1}^{V-1} u_{v}\right) \prod_{v=1}^{V-1}\left(\Delta_{n}\left(u_{v}\right) \frac{d u_{v}}{2 \pi}\right) h\left(u_{1}, \ldots, u_{V-1}\right) .
$$

where

$$
h\left(u_{v}, v=1, \ldots, V-1\right):=\int \prod_{e=1}^{E} f^{(e)}\left(\chi_{e}\right) \prod_{c=1}^{C} d y_{c},
$$

and, by (3.4),

$$
\chi_{e}=\sum_{c=1}^{C} y_{c}[c: e]+\sum_{v=1}^{V-1} u_{v} N_{v, e}=X_{e}+\sum_{v=1}^{V-1} u_{v} N_{v, e} .
$$

It follows from (1.7) and Theorem 1 that $h$ is well defined. Note also that $h(0, \ldots, 0)=I(G)$, as defined in (1.8). To end the proof, we need to notice two more facts:

(a) the measure $\frac{1}{n} \Delta_{n}\left(-\sum_{v=1}^{V-1} u_{v}\right) \prod_{v=1}^{V-1}\left(\Delta_{n}\left(u_{v}\right) d u_{v} / 2 \pi\right)$ converges weakly to the measure $\delta_{\{0\}}$, since the Fourier coefficients converge (see [F2, Lemma 7.1]), and moreover the measures have variations uniformly bounded.

Indeed, by Theorem 1 , with $p^{-1}=Z=(V-1) / V$, we get

$$
\begin{aligned}
\frac{1}{n} \int \mid \Delta_{n} & \left(-\sum_{v=1}^{V-1} u_{v}\right) \prod_{v=1}^{V-1} \Delta_{v}\left(u_{v}\right) \mid \prod_{v=1}^{V-1} d u_{v} / 2 \pi \\
& \leq \frac{1}{n}\left\|\Delta_{n}\right\|_{p}^{V}=\frac{1}{n}\left(O\left(n^{1 / V}\right)\right)^{V}=O(1) \quad\left(\text { since }\left\|\Delta_{n}\right\|_{p}=O\left(n^{1-1 / p}\right)\right) .
\end{aligned}
$$

(b) $h$ is continuous in the $u_{v}$.

Indeed, by Theorem 1 , the conditions $(1.7)$ and the fact that the matrix $M^{*}$ has integer entries ensure that

$$
|I(G)| \leq \prod_{e=1}^{E}\left\|f^{(e)}\right\|_{p_{e}}
$$

and thus the functional $I: \prod_{e=1}^{E} L_{p_{e}} \longrightarrow \mathbf{R}$ is continuous. But $h$ is the composition of the continuous function $I$ with the continuous functionals $T_{e}: \mathbf{R}^{V-1} \rightarrow$ $\mathscr{L}_{p_{e}}$ defined by $T_{e}\left(u_{v}, v=1, \ldots, V-1\right)=f^{(e)}\left(.+\sum_{v} u_{v}(v: e)\right)$ (here, $f^{(e)}$ are fixed), and thus is continuous. (The functionals $T_{e}$ are clearly continuous when $f^{(e)}$ is a continuous function on $(-\pi, \pi)$; and since a function in $\mathscr{L}_{p}$ can be approximated in $\mathscr{L}_{p}$ sense by continuous functions (recall that $\mathscr{L}_{\infty}$ is in our notation actually $C$ ), the same follows for any function in $\mathscr{L}_{p_{e}}$.)

By (3.5), and facts (a), (b) above, we get:

$$
\frac{S_{n}}{n} \longrightarrow h(0, \ldots, 0)=\int \prod_{e=1}^{E} f^{(e)}\left(X_{e}\right) \prod_{c=1}^{C} \frac{d y_{c}}{2 \pi} \triangleq I(G) .
$$


Remark. As noted already in $\S 1$, the dependence structure given by (1.5) is the bond matroid of the graph, independently of the particular choice of the set of "cycle" coordinates. Furthermore, $I(G)$ is also independent of the choice of the coordinates $y_{c}$, since changing the basis of fundamental cycles amounts to a linear change of variables with integer coefficients (see [N, Theorem 2.19]). Thus, the result is independent of the initial choice of the tree $T$.

\section{ACKNOWLEDGMENT}

The proof the Theorem 2 is accomplished by using ideas from [F2], with an elegant improvement due to Herold Dehling, for which we thank him.

\section{REFERENCES}

[A] F. Avram, On bilinear forms in Gaussian random variables, Toeplitz matrices and Parseval's relation, preprint, 1986.

[A2] __ Asymptotics of sums with dependent indices and convergence to the Gaussian distribution, preprint, 1987.

[BL] J. Bergh and J. Lofstrom Interpolation spaces, Springer-Verlag, New York, 1976.

[BM] J. Bondy and U. Murty, Graph theory with applications, North Holland, New York, 1976.

[BP] V. Bryant and H. Perfect, Independence theory in combinatorics, Chapman and Hall, New York, 1980.

[D] H. Dehling, private communication.

[F1] R. Fox and M. Taqqu, Non-central limit theorems for quadratic forms in random variables having long range dependence, Ann. of Prob. 13 (1985), 428-446.

[F2] _ Central limit theorems in random variables having long range dependence, Probab. Th. Rel. Fields 74 (1987), 213-240.

[GS] V. Grenander and G. Szego, Toeplitz forms and their application, Univ. California Press, Berkeley, 1958.

[G1] L. Giraitis and D. Surgailis, C.L.T. theorems and other limit theorems for functionals of Gaussian processes, Z. Wahr. verw. Geb. 70 (1985), 191-212.

[G2] __ Multivariate Appell polynomials and the central limit theorem, in: Dependence in Probability and Statistics, E. Eberlein and M. Taqqu, eds., Birkhauser, Boston, 1986.

[K] Y. Katznelson, An introduction to harmonic analysis, Wiley, New York, 1968.

[LZ] J. H. Lowenstein and W. Zimmermann, The power counting theorem for Feynman integrals with massless propagators, Comm. Math. Phys. 44 (1975), 73-86.

[LC] W. Light and E. Cheney, Approximation theory in tensor product spaces, Lect. Notes in Math. 1169, Springer-Verlag, New York, 1980.

[M] E. Manoukian, Renormalization, Academic Press, New York, 1983.

[N] N. Nakanishi, Graph theory and Feynman integrals, Gordon and Breach Science Publishers, New York, 1971.

[Wg] S. Weinberg, High energy behavior in quantum field theory, Phys. Rev. 118 (1960), 838.

[W] D. Welsh, Matroid theory, Academic Press, New York, 1976.

Mathematics Department, 521 Lake Hall, Northeastern University, Boston, MASSACHUSETTS 02115 